\title{
DRIVER BEHAVIOR IN OVERTAKING ACCIDENTS AS A FUNCTION OF DRIVER AGE, ROAD CAPACITY AND VEHICLE SPEED: A CASE STUDY IN IRAQ
}

\author{
Husam Muslim ${ }^{1}$, Makoto Itoh ${ }^{2}$ \\ ${ }^{1}$ Graduate School of Systems and Information Engineering, Risk Engineering Department \\ ${ }^{2}$ Faculty of Engineering, Information and Systems \\ ${ }^{12}$ University of Tsukuba, Tsukuba, Ibaraki, Japan \\ Email: hussam@css.risk.tsukuba.ac.jp
}

\begin{abstract}
Summary: Overtaking accidents are one of the most serious types of road traffic accidents in terms of personal injury. They are mostly caused by drivers' misjudgment of the situation due to human factors (e.g., limited skills and/or information processing abilities), speeding, roadway capacity, and weather condition. Although the overtaking maneuver can be necessary, drivers' use of it is often not justified. The present study aims to identify factors affecting driver behavior in critical overtaking maneuvers. The study collected and analyzed 4,902 real overtaking accidents that occurred on highways in Iraq for the years 20052016. Three categories of overtaking accidents were extracted and analyzed in terms of driver age, road capacity (number of lanes and curvature), and vehicles type and speed: 1) head-on collisions that occur when the overtaking vehicle strikes the front-end of an oncoming vehicle; 2) rear-end collisions that occur when the overtaking vehicle strikes the rear-end of a vehicle ahead; and 3) side-impact collisions that occur when the overtaking vehicle strikes or is struck by a vehicle in the adjacent lane. The results indicate that number and types of overtaking accidents were significantly affected by driver age, while the tendency of drivers to overtake was influenced by the type of leading vehicles. A significant interaction between driver age and road capacity was detected in terms of higher accident rates. Correlations between overtaking accidents types, road curvature, and overtaking speed were also identified. The findings have implications for the future design of drivers assistance systems.
\end{abstract}

\section{INTRODUCTION}

Overtaking is one of the most dangerous maneuvers frequently engaged in by drivers (Clarke et al., 1999). It is a dynamic and complicated driving task involving steering and speed controls, which requires high driver attention to monitor surrounding traffic and predict potential opportunities for time-critical decision making and action implementation (Bar-Gera and Shinar, 2005). The overtaking drivers need to continuously predict potential hazards in the current and targeted lanes, plan their next safe path, and perform the plan while continuing to scan the surrounding environment and readily respond to unpredictable hazards (Gray and Regan, 2005). Performing such tactical maneuvers tends to increase the likelihood of driver errors, which may result in sever traffic accidents. For example, nearly $75 \%$ of road traffic accidents caused by lateral maneuvers involve driver failure to identify hazards (Knipling, 1993). While risky in terms of accidents occurrence likelihood, overtaking is necessary in some cases, and roadways are supposed to be designed to accommodate multiple vehicles with different speed. As overtaking maneuvers cannot be avoided, driver performance should be improved to prevent accidents and realize safer driving environments. In order to recommend accident solutions, this 
study set out to identify aspects with critical impact on cognitive factors in overtaking, such as expectancy, prediction, and risk response.

Overtaking is often related to variations in speed and drivers' desire to minimize the traveling time and avoiding slow traffic. Overtaking may be performed in the interests of safety, such as avoiding rapidly decelerating or stopped vehicles (Bar-Gera and Shinar, 2005; Vlahogianni, 2013). It can also be motivated by drivers' individual behavior, e.g. feelings of competitiveness, thrill seeking, and aggressiveness (Farah et al., 2008). As the driving environment is highly complex and dynamic, overtaking maneuvers may occur for other reasons.

Utilized driving simulators and field experiments, the impact of human, vehicular, and environmental factors on overtaking drivers' behavior and performance has been investigated and discussed in the literature. For example, studies have investigated the effects of driver age, speed variability, and type of the vehicle ahead (Bar-Gera and Shinar, 2005; Rajalin et al., 1997). Other researchers have focused on overtaking driver behavior by modeling driver sight, investigating overtaking duration, and determining speed differential threshold, distance gap between vehicles, and the lateral displacement of the overtaking vehicle (Vlahogianni, 2013; Haneen and Tomer, 2010; Brown and Hummer, 2000). Findings of these studies have contributed to help designers and engineers to enhance vehicle capabilities, develop in-vehicle protective devices, and improve road structure and capacity (Hegeman et al., 2009). What is not yet clear is how these factors are related to overtaking accidents in real-world driving.

While driving simulators and field experiments are safe, reliable, and efficient approaches, drivers' behavior may differ from their real-world behavior (Santos et al., 2005). An alternative and well established approach in road traffic safety is the use of quantitative case studies. Few studies have investigated real accidents data, mainly because it is difficult to collect sufficient accurate information about each accident that can be reliably used to validate the findings and draw conclusions.

To explore parameters for the future development of driver assistive technologies, the present study aims at providing a better understanding of the evasive actions taken by the drivers during real-world overtaking. The present study collected and analyzed detailed information describing real overtaking accidents that occurred on Iraqi highways. The first objective was to understand the behavior of the overtaking driver and the mechanism of overtaking accidents. The second objective was to identify contributory factors that impact the overtaking accident rates.

\section{METHOD}

\section{Data Collection}

During the past ten years, Iraq has witnessed approximately 70,000 road-accidents with approximately 30,000 fatalities and more than 80,000 injuries (TPA, 2016). The highest percent (75\%) of the overall collected accidents occurred on main roadways (rural and urban highways, freeways, and border roads). Highway accidents account for $49 \%$ of all road-accidents and $65 \%$ of main roadway accidents. A high percentage (46\%) of highway accidents was caused by drivers performing critical overtaking maneuvers at high speed. Overtaking accidents on highways are, thus, a particularly serious problem, and were, therefore, selected for analysis. A total of 4,902 real-world overtaking accidents were examined. Each case was summarized as a 
database entry containing the main objective features (e.g. information relating to vehicles involved, type of road, and speed), a sketch plan, and an explanation of causal factors.

\section{Definitions of Terms Used in This Study}

Two-lane Highway (TLH): This type of roadway comprises one lane in each direction with no median barrier to separate between lanes (AASHTO, 2004), as shown in Figure 1.

Two-way Highway (TWH): This type of roadway comprises one or two lanes in each direction with median barrier to separate between directions (AASHTO, 2004), as shown in Figure 2.

Overtaking Maneuver (OM): Overtaking occurs when a driver maneuvers a vehicle laterally from one lane to another, then longitudinally to pass front vehicles, and then laterally to return to the initial lane (Jenkins and Rilett, 2005). Hence, OM comprises three sequential parts: lane changing, passing, and lane returning (Figures 1 and 2). The maneuver starts when a driver of a subject vehicle (SV) first changes lanes from the host lane (HL) to a destination lane, which can be an adjacent lane (AL) with the same direction of travel or an oncoming lane (OL) with an opposite direction of travel. Vehicles located in AL are referred to as adjacent vehicles (AV) and vehicles located in OL are referred to as oncoming vehicles (OV). The second part starts once the driver reaches the destination lane and the vehicle is driving straight forward to pass one or more leading vehicles (LV) that are located in HL. The third part begins when the driver changes lanes from the destination lane to HL. The overtaking maneuver ends once the SV's driver returns completely to $\mathrm{HL}$ and starts driving straight forward again.

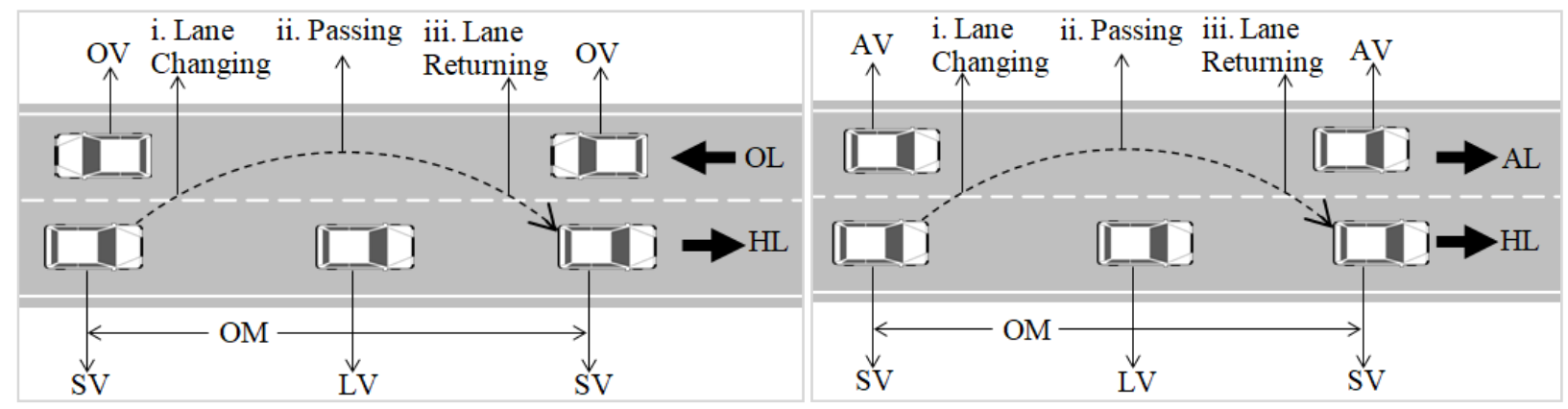

Figure 1. Overtaking manoeuvre on TLH

Figure 2. Overtaking manoeuvre on TWH

\section{Types of Overtaking Accidents}

Head-on Collisions (HC). A head-on collision occurs when the front ends of two vehicles that are traveling towards each other collide. The risk of head-on collisions increases when a driver initiates an overtaking on TLH type. In this study, the investigated HC occurred when a subject vehicle (SV) initiated an overtaking on TLH and stroke an OV in OL (Figure 3). This type of collisions accounts for nearly 39.4\% (1934 accidents) of all investigated overtaking accidents. A large portion $(86 \%)$ of the investigated $\mathrm{HC}$ were fatal crashes, which emphasizes the seriousness of head-on collisions, particularly when overtaking in high speed.

Rear-end Collisions $(R C)$. Rear-end collisions are a frequent type of road accidents in which a vehicle runs into the rear end of a preceding vehicle. This study reports 1,058 rear-end collisions occurred during the process of overtaking. For TLH type, an RC occurred between OV and other 
preceding vehicles in the original lane due to decreasing headway or critical spacing between vehicles (Figure 3). For TWH type, in addition to RC between overtaking and preceding vehicles, $\mathrm{RC}$ occurred between $\mathrm{OV}$ and AV during the passing phase (Figure 4).

Side-impact Collisions (SC). This type of traffic collisions occurs when a vehicle's side is impacted by another vehicle during intersection crossing, lane changing/merging/departing and overtaking. Two types of SC were distinguished. One involves vehicles that are traveling in opposite directions when an OV strikes the side of SV, while SV is attempting to return to its original lane (Figure 3). The other type involves vehicles that are travelling in the same direction when an SV sideswipes or cuts in front of an adjacent or preceding vehicle during lane changing and lane returning respectively (Figure 4).

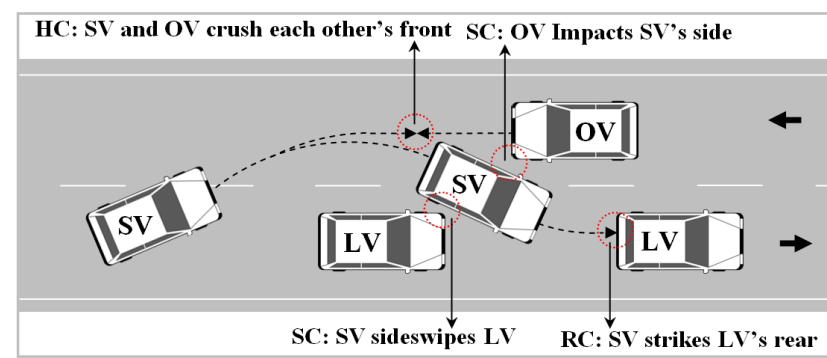

Figure 3. Types of overtaking accidents on TLH

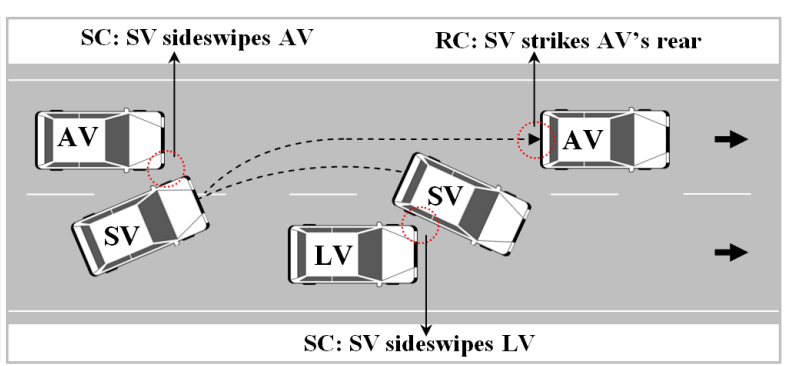

Figure 4. Types of overtaking accidents on TWH

\section{RESULTS AND DISCUSSIONS}

According to Traffic Police Administration in Iraq, in approximately $80 \%$ of highway accidents, driver error was the major cause. This percentage is slightly more than that recognized by other researches (Knipling, 1993). Thus, understanding why drivers are most likely to be at fault in overtaking can help in reducing overtaking accidents and lead to a better understanding of overtaking driver behavior. Although age is not itself an error or impairment, the role of age is critical in driver behaviour and performance. The drivers involved in overtaking accidents were categorized into three age groups: young drivers (YD) between 18-40 years, middle-aged drivers (MD) between 41-60 years, and older drivers (OD) above 60 years (WHO, 2016). Table 1 shows the percentage of each age group involvement in overtaking accidents. The highest number of collisions was caused by young drivers followed by middle-aged drivers with slight difference. The distribution of overtaking accidents on the range of drivers age indicates the negative association between drivers age and accident rates $(r=-0.301, p<0.01)$, as shown Figure 5 .

Table 1. Age-related percentage of overtaking accidents

\begin{tabular}{ccc}
\hline $\begin{array}{c}\text { Age } \\
\text { groups }\end{array}$ & $\begin{array}{c}\text { Overtaking } \\
\text { accidents }\end{array}$ & Percentage \\
\hline YD & 2402 & $49 \%$ \\
\hline MD & 2206 & $45 \%$ \\
\hline OD & 294 & $6 \%$ \\
\hline
\end{tabular}

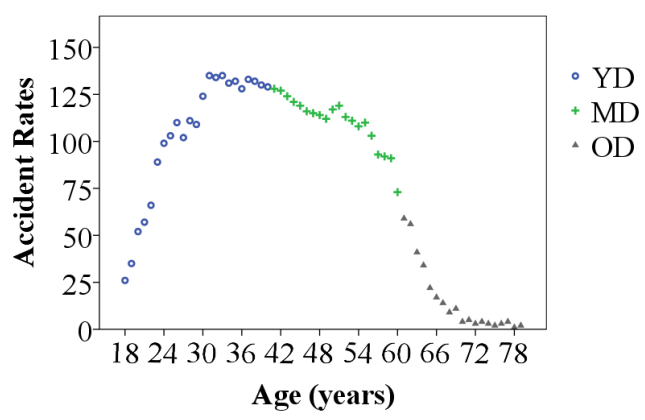

Figure 5. Overtaking accident rates as a function of driver age

Although the effects of age on driver performance during the process of overtaking have been investigated by several researchers, very little is known about how driver age influences the type 
of overtaking accident. Figure 6 compares the three types of overtaking accidents between age groups. The Chi-square test revealed significant differences between age groups for each type of accident $\left(\chi^{2}(3)=232.08, p<0.01\right)$. A closer inspection of the figure shows that the young drivers were more prone to $\mathrm{HC}$ and $\mathrm{SC}$, which may indicate that young drivers tend to make a faulty overtaking decision-e.g., overtaking in critical gaps or misjudging the speed of the oncoming traffic. The high percentage of YD involvement in side-impact collisions may also indicate that young drivers are less attentive to the adjacent traffic during lane changing. For MD group, the percentages of drivers' involvement in each type of overtaking accidents were comparable. The results of OD group depict that they are more cautious about overtaking gap, but less attentive to the adjacent traffic stream. These results suggest that the drivers of each age group are prone to a certain dangerous situation which may require a different type of driving support.

Highway capacity can also play a significant role in driving task complexity and the likelihood of accident occurrence. Figure 7 presents overtaking accidents that was collected from urban and rural highways with a total length of nearly $26,200 \mathrm{~km}$ comprising $14,033 \mathrm{~km}$ of TLH and $12,167 \mathrm{~km}$ of TWH. Road curvature, straight and curved sections, was also taken into consideration due to the potential effect on passing sight and duration. Number of collisions were significantly different between highway types $\left(\chi^{2}(3)=1462.97, p<0.01\right)$. The large difference in the number of HC between TLH and TWH is because when the drivers initiate an overtaking on TLH type, they are occupying an opposing direction lane, while the drivers can overtake on TWH type using an adjacent lane with the same direction of travel. In terms of road curvature, the test revealed a significant difference in accident rates $\left(\chi^{2}(2)=1498.43, p<0.01\right)$. The higher number of HC in the curved-TLH sections might indicate that road curvature have a negative influence on drivers' perception leading to increase the risk of overtaking. The high number of $\mathrm{HC}$ in the straight-TLH might indicate that straight and open roadway sections may encourage the drivers to increase the speed and overtake. Figure 8 shows how driver age and highway capacity combine to affect accident rates, which were significantly different between age groups for each type of highway $\left(\chi^{2}(3)=331.23, p<0.01\right)$. A negative correlation was indicated between driver age and road capacity $(r=-0.397, p<0.01)$. These results suggest that the young drivers are showing the worst risk taking behavior compared to other age groups.

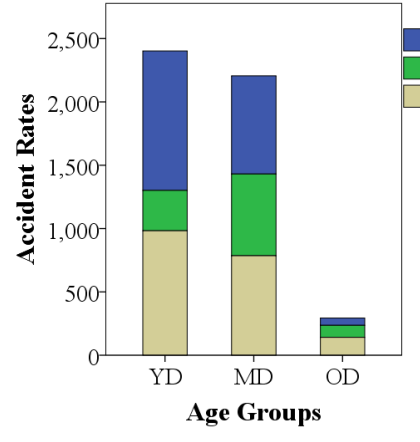

Figure 6. Overtaking accident rates as a function of age groups

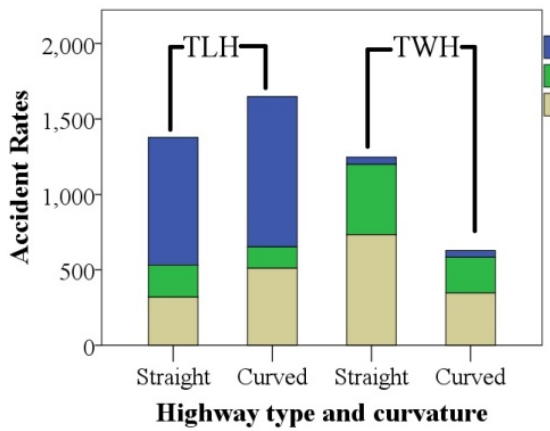

Figure 7. Overtaking accident rates as a function of highway type and curvature

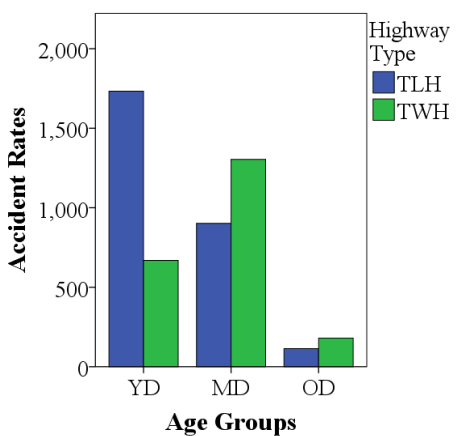

Figure 8. Impact of the interaction between driver age and highway type on overtaking accident rates

The study has also investigated the effects of the vehicles ahead to be overtaken and the intended overtaking speed on accident rates, as shown in Table 2. Types of leading vehicles were standardized into three categories: light car, truck, and trailer. It would be more difficult to calculate the exact speed of all vehicles involved in real-world accidents due to lack of traffic 
monitoring tools compared to controlled experimental studies. However, the speed of the subject vehicle was calculated according to the site information, such as the skid marks on the road surface, crash impact and testimonies. A significant association between types of leading vehicle and types of accidents was detected $\left(\chi^{2}(3)=62.63, p<0.01\right)$. This highlights the influence of leading vehicle on drivers' cognitive action and the tendency to overtake. The reason may be attributed to the effect of vehicle size on drivers' sight, risk feeling, and the overtaking duration. A significant association between the overtaking speed and type of overtaking accidents was also detected $\left(\chi^{2}(3)=757.62, p<0.01\right)$. The highest rate of $\mathrm{HC}$ occurred when the $\mathrm{SV}$ speed was between $100-120 \mathrm{~km} / \mathrm{h}$ followed by SC when the speed was between $80-100 \mathrm{~km} / \mathrm{h}$. Although a previous study reported that some drivers tend to overtake vehicles that were traveling faster than their average speed (Bar-Gera and Shinar, 2005), it was difficult to determine the speed difference of SV before and after the initiation of the overtaking in real-world investigations.

Table 2. Distribution of overtaking accidents according to types of leading vehicle and overtaking speed

\begin{tabular}{|c|c|c|c|c|c|c|}
\hline \multirow[b]{2}{*}{ Type of LV } & \multirow{2}{*}{$\begin{array}{c}\text { Accident } \\
\text { type }\end{array}$} & \multicolumn{4}{|c|}{ Estimated Overtaking Speed $(\mathrm{km} / \mathrm{h})$} & \multirow[b]{2}{*}{ Total } \\
\hline & & $60-80$ & $80-100$ & $100-120$ & $>120$ & \\
\hline \multirow{3}{*}{ Light car } & $\mathrm{HC}$ & 5180 & 76 & 137 & 183 & 447 \\
\hline & $\mathrm{RC}$ & 27 & 91 & 113 & 115 & 346 \\
\hline & $\mathrm{SC}$ & 139 & 177 & 102 & 81 & 499 \\
\hline \multirow{3}{*}{ Truck } & $\mathrm{HC}$ & 54 & 133 & 134 & 192 & 513 \\
\hline & $\mathrm{RC}$ & 31 & 36 & 85 & 59 & 211 \\
\hline & $\mathrm{SC}$ & 63 & 174 & 201 & 145 & 583 \\
\hline \multirow{3}{*}{ Trailer } & $\mathrm{HC}$ & 60 & 233 & 392 & 289 & 974 \\
\hline & $\mathrm{RC}$ & 33 & 112 & 214 & 142 & 501 \\
\hline & $\mathrm{SC}$ & 94 & 306 & 241 & 187 & 828 \\
\hline Total & & 552 & 1,338 & 1,619 & 1,393 & 4,902 \\
\hline
\end{tabular}

\section{CONCLUSIONS}

This study used real road-accidents to investigate how driver behavior in overtaking maneuvers is effected by driver age, roadway, and vehicular factors. The study has shown that overtaking, which occurs when the drivers decide to pass other vehicles, is a critical maneuver with very serious consequences for roadways safety. Accidents were most serious on highways where overtaking is permitted but that were lacking in terms of traffic regulation and infrastructure. In addition to an association between driver age and the number of overtaking accidents, each age group was prone to a distinct type of overtaking accidents. Highway structure and curvature revealed no significant effect on the overtaking accident rates. However, the interaction between driver age and highway types had a significant effect on driver behavior and safety. The tendency of drivers to overtake was also significantly influenced by driver age and type of leading vehicles. The study has found that the overtaking accident rates were also affected by the overtaking speed. These results indicate that drivers may initiate overtaking even when road section is dangerous and overtaking sight is insufficient or impaired.

The present study highlights the need for enhancing cognitive factors in driving to avoid drivers' misjudgment in overtaking. Furthermore, the high overtaking accident rates during the passing stage suggest that supporting perception-action time of drivers should also be made available. These findings have implications for the development of driver assistive technologies. A limitation of this study is the absence of traffic monitoring tools, thus, further controlled studies using driving simulators and field data, are, therefore, needed to validate these findings. 


\section{ACKNOWLEDGMENTS}

The authors wish to acknowledge the Iraqi Traffic Police Administration for providing all accident data and for their timely help in analyzing the large number of samples.

\section{REFERENCES}

AASHTO (2004). A Policy on Geometric Design of Highways and Streets. Washington, DC.

Bar-Gera, H., Shinar, D. (2005). The tendency of drivers to pass other vehicles. Transportation Research Part F: Traffic Psychology and Behaviour, vol. 8, 429-439.

Brown, R., \& Hummer, J. (2000). Determining the best method for measuring no-passing zones. Transportation Research Record: Journal of the Transportation Research Board, vol. 1701, 61-67.

Clarke, D. D., Ward, P. J., \& Jones, J. (1999). Processes and countermeasures in overtaking road accidents. Ergonomics, 42(6), 846-867.

Farah, H., Yechiam, E., Bekhor, S., Toledo, T., \& Polus, A. (2008). Association of risk proneness in overtaking maneuvers with impaired decision making. Transportation research part F: traffic psychology and behaviour, 11(5), 313-323.

Gray, R., Regan, D. M. (2005). Perceptual processes used by drivers during overtaking in a driving simulator. Human Factors, 47(2), 394-417.

Haneen, F., \& Tomer, T. (2010). Passing behavior on two-lane highways. Transportation Research Part F: Traffic Psychology and Behaviour, 13(6), 355-364.

Hegeman, G., Tapani, A., \& Hoogendoorn, S. (2009). Overtaking assistant assessment using traffic simulation. Transportation research part C: emerging technologies, 17(6), 617-630.

Jenkins, J. M., Rilett, L. R. (2005). Classifying Passing Maneuvers A Behavioral Approach. Transportation Research Record: Journal of the Transportation Research Board, No. 1937, Transportation Research Board of the National Academies, Washington, D.C., 14-21.

Knipling, R. R. (1993). IVHS technologies applied to collision avoidance: Perspectives on six target crash types and countermeasures. Proceedings of the 1993 Annual Meeting of IVHS America: Surface Transp.: Mobility, Technology, and Society, 249-259.

Rajalin, S., Hassel, S. O., Summala, H. (1997). Close-following drivers on two-lane highways. Accident Analysis and Prevention, 29(6), 723-729.

Santos, J., Merat, N., Mouta, S., Brookhuis, K., \& De Waard, D. (2005). The interaction between driving and in-vehicle information systems: Comparison of results from laboratory, simulator and real-world studies. Transportation Research Part F: Traffic Psychology and Behaviour, 8(2), 135-146.

Traffic Police Administration (TPA). (2016). Road Traffic Accidents in Iraq: Final Report. Baghdad: Department of Traffic.

Vlahogianni, E. I. (2013). Modeling duration of overtaking in two lane highways. Transportation research part F: traffic psychology and behaviour, vol. 20, 135-146.

World Health Organization (WHO). (2016). World health statistics 2016: monitoring health for the SDGs sustainable development goals. Geneva: World Health Organization. 